\title{
Resolving the ionized wind of the post-red supergiant IRC +10420 with VLTI/AMBER ${ }^{\star}$
}

\author{
W. J. de Wit ${ }^{1}$, R. D. Oudmaijer ${ }^{1}$, M. A. T. Groenewegen ${ }^{2}$, M. G. Hoare ${ }^{1}$, and F. Malbet ${ }^{3}$ \\ 1 School of Physics \& Astronomy, University of Leeds, Woodhouse Lane, Leeds LS2 9JT, UK \\ e-mail: w.j.m.dewit@leeds.ac.uk \\ 2 Institute for Astronomy, University of Leuven, Celestijnenlaan 200D, 3001 Leuven, Belgium \\ ${ }^{3}$ Laboratoire d'Astrophysique, Observatoire de Grenoble, BP 53, 38041 Grenoble Cedex 9, France
}

Received 1 October 2007 / Accepted 29 November 2007

\section{ABSTRACT}

\begin{abstract}
Aims. The paper investigates the milli-arcsecond scale structure of the present-day wind of the post-red supergiant IRC +10420 . Methods. We use three telescopes of the VLT Interferometer in combination with the AMBER near-infrared beam combiner to measure spectrally dispersed correlated fluxes in the $K$-band around the $\mathrm{Br} \gamma$ transition. The resulting visibilities are compared with predictions of various simple models in order to infer the size of the observed emission region.

Results. The Br $\gamma$ line is resolved by VLTI+AMBER on all three baselines, with the maximum projected baseline extending $69 \mathrm{~m}$ and a PA ranging between $10^{\circ}$ and $30^{\circ}$. A differential phase between line and continuum is detected on the longest baseline. The best fitting model, a Gaussian flux distribution, implies a size of the $\mathrm{Br} \gamma$ emission region of 3.3 milli-arcsec $(F W H M)$. The size of the continuum emission is not constrained by the observations. From a comparison of the AMBER equivalent width of Br $\gamma$ with measurements from various epochs, we find that the stellar photosphere contributes about $60 \%$ of the total continuum light at $2.2 \mu \mathrm{m}$. The remaining $40 \%$ continuum emission originates from structures larger than the 66 mas AMBER field of view. This independently confirms similar results made by previous studies. If the $\mathrm{Br} \gamma$ emission is optically thin, then the observations do not allow to make any inferences about the shape of the line forming region. However, there is indirect evidence that the hydrogen recombination line emission is optically thick. In that case, using simple arguments, we find that the line emitting region is elongated. This is because the spectrum indicates that the projected line emitting area is about twice that of the stellar surface. A circular Br $\gamma$ line emitting area, however, would have a size an order of magnitude larger using the size of the emission region measured along our baseline. We briefly mention the possibilities whether such a structure is due to a bi-polar flow or a circumstellar disk.
\end{abstract}

Key words. stars: evolution - stars: mass-loss - supergiants - stars: individual: IRC +10420 - techniques: interferometric

\section{Introduction}

Throughout the evolution of a high-mass star, stellar winds carry off a significant fraction of the initial star mass. This process dominates their evolution and determines their final fate. In the late stages of massive star evolution, important information about the mass loss process is contained in the complicated circumstellar nebulae that are, for example, found around LBVs and Wolf-Rayet stars (Nota et al. 1995; Nota \& Clampin 1997; Van der Sluys \& Lamers 2003). The nebulae provide information about the mass-loss history; they allow us to identify phases where stellar wind properties (like the wind momentum, geometry and mass loss) vary, sometimes considerably. One explanation for the observed asymmetries in these nebulae concerns the collision between a recent, fast wind with the slower wind from a previous mass losing phase. Importantly, at least one of these winds should be asymmetric to yield an aspherical nebula. This could possibly be due to mass loss anisotropies at the stellar surface (Maeder 2002). During which evolutionary phase this would actually occur is not known however, and various ideas have been put forward (see e.g. Mellema 1997; Heger \& Langer 1998; Dwarkadas \& Owocki 2002).

IRC +10420 is one of the few stars that are known to evolve from the Red Supergiant (RSG) phase back towards the blue (see

^ Based on observations with VLTI, proposal 077.D-0388. e.g. the review by Oudmaijer et al. 2008). IRC +10420 underwent an extremely rapid increase in its surface temperature of $\sim 2200 \mathrm{~K}$ over 30 years and currently has a mid-A spectral type (see Oudmaijer et al. 1996; Klochkova et al. 2002). Its estimated distance of 3.5 to $5 \mathrm{kpc}$ implies a luminosity typical for a star with an initial mass of around $40 M_{\odot}$, which places it close to the Humphreys-Davidson limit in the Hertzsprung-Russell diagram (Jones et al. 1993). The star is bright at $2.2 \mu \mathrm{m}\left(K_{\mathrm{s}}=3.6\right)$ and exhibits a huge infrared excess due to warm dust indicating that it was only recently in an extreme mass-losing Red Supergiant phase (Oudmaijer et al. 1996). These properties make the star a prime candidate to eventually become a Wolf-Rayet star (Jones et al. 1993).

The circumstellar environment of IRC +10420 has various distinctive components, and we mention the basic features on the various size scales below. From HST images it is found that IRC +10420 's optical reflection nebula portrays a spherical geometry at scales from $\sim 2^{\prime \prime}$ out to 6 to $8^{\prime \prime}$ (Humphreys et al. 1997). This round appearance is also seen at other wavelengths at these scales, for example in the $J$-band by Kastner \& Weintraub (1995), and in CO rotational transitions by Castro-Carrizo et al. (2007). On a scale of about 1", a bipolar geometry, with two patches of $K$-band and mid-IR emission to the $\mathrm{NE}$ and SW direction of the central object is visible (Humphreys et al. 1997). Given the relatively large distance from the star, this emission should be scattered radiation rather than thermal 
emission from hot dust. The location of these patches corresponds to an approximate position angle of $40^{\circ}$. Closer in and down to $0.3^{\prime \prime}$, HST reveals a complicated and non-uniform distribution of various jet-like, ray and arc features (Humphreys et al. 1997).

At even smaller angular scales, the dusty environment responsible for the near-IR excess has been resolved with speckle interferometry at $2.11 \mu \mathrm{m}$ (Blöcker et al. 1999). Modelling the spectral energy distribution (SED) and visibility curves with DUSTY leads these authors to conclude that IRC +10420 is surrounded by two separate spherical shells with diameters of $0.070^{\prime \prime}$ and $0.310^{\prime \prime}$ centred on the star. Finally, the geometry on a size scale comparable to the stellar radius $(\sim$ milli-arcsec $)$ can be probed with the various emission lines, like $\mathrm{H} \alpha$ and Fe II, that are reflected off the large scale blue nebula (Humphreys et al. 2002; Davies et al. 2007). From IFU observations of the nebula, Davies et al. find evidence for a present-day wind which has an axi-symmetric, rather than spherically symmetric geometry. The $\mathrm{H} \alpha$ emission symmetry axis is found to have a position angle of $33^{\circ}$.

In this paper on IRC +10420 we present complementary observations of the subarcsecond ionised and continuum emitting material inside the 70 mas dust shell found by Blöcker et al. (1999). They found that about $60 \%$ of the $K$-band continuum emission remains unresolved (see also Monnier et al. 2004), and this emission is presumably photospheric radiation from the star itself. We use the VLT interferometer in conjunction with the near-IR three telescope beam combiner AMBER, attaining a maximum spatial resolution of 6.5 mas. Our ultimate objective is to measure the size and shape of the $\mathrm{Br} \gamma$ emission line region, which gives a direct measurement of the present-day mass-loss geometry, an important ingredient to the mass-loss puzzle. In Sect. 2 we present the AMBER data, and provide a list of steps and considerations taken in the reduction process. Results regarding the final visibility, flux and differential phase spectra of IRC +10420 are presented in Sect. 3. In this section we derive the size for the continuum and $\mathrm{Br} \gamma$ emission. We discuss the shape of the Br $\gamma$ emission region in Sect. 4, where based on simple considerations, we suggest it is elongated along the VLTI baseline of the observation with a PA of $\sim 20^{\circ}$. We conclude in Sect. 5.

\section{Observations and data reduction}

\subsection{AMBER data reduction: general considerations}

AMBER is the VLTI's near infrared, three telescope beam combiner (Petrov et al. 2007). The interferometric beam is fed into a spectrograph delivering a spectrally dispersed interferogram that is spatially encoded on a detector. AMBER can operate with two or three telescopes, and offers three spectral resolutions. The AMBER data reduction is not (yet) a straightforward process and particular care should be taken in reducing and analysing the data.

The reduction of AMBER data can be split in two distinctive steps. Ever since the discovery of an electronic interference pattern on the detector, a first reduction step must involve a digital filtering process that needs to be applied to all data taken with AMBER (Li Causi et al. 2008). A software routine by the name of amdc has been developed that identifies the electronic interference frequencies in Fourier space by means of a dark frame, and creates a "Fourier mask". This mask needs to be applied to the data, and amdc replaces the power at the corrupted

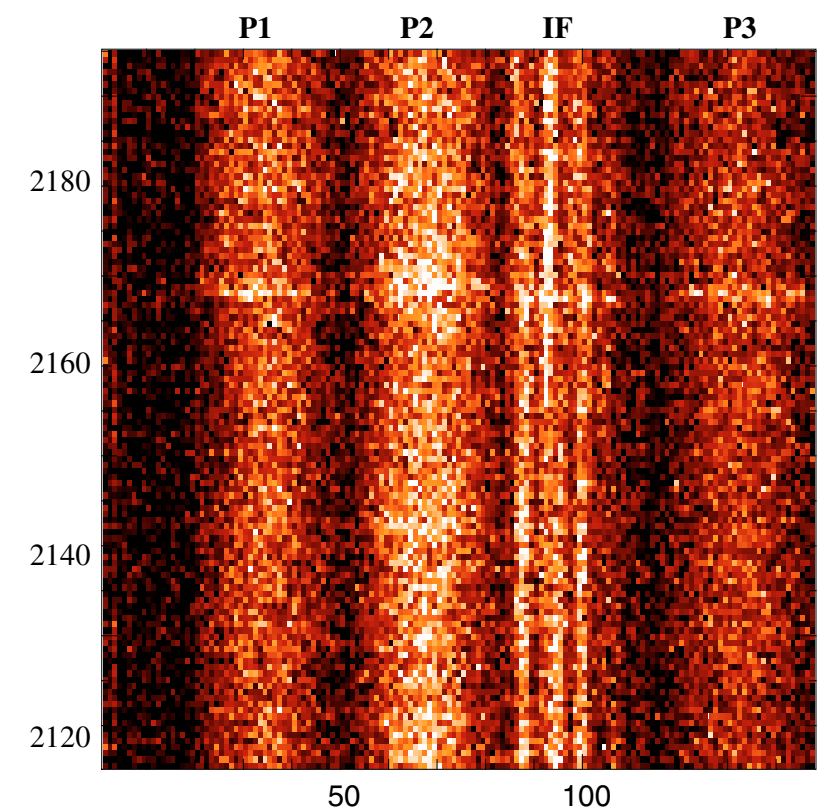

Fig. 1. Illustration of an AMBER frame of the target star IRC +10420 showing fringes. An approximate wavelength scale in $\mathrm{nm}$ is given along the $y$-axis, pixels along $x$-axis. The frame gives the three telescope channels (P1, P2, P3), and the interferometric channel (IF). The Br $\gamma$ emission line can be identified as the horizontal enhancement in recorded ADUs.

frequencies with a new value based on the power at the neighbouring frequencies.

The second step consists of converting the Fourier filtered AMBER data to (raw) visibilities. This step can be done using the dedicated AMBER software amdlib written by the consortium (see Tatulli et al. 2007b, for an in depth explanation). In short, the software algorithm relies on a mathematical description of the interferogram, with empirical input from the calibration and alignment unit of the AMBER instrument. It records VLTI+AMBER instrumental characteristics at the beginning of each night, these are used to create the so-called pixel-to-visibility matrix (P2VM). The P2VM then converts the observed science target fringe patterns into a measurement of coherent flux. The photometric flux spectra for each telescope are recorded simultaneously with the interferogram. An example of an AMBER exposure (or frame) of IRC +10420 is given in Fig. 1. The data reduction package calculates the interferometry observables. In the case of three telescopes, these are the three squared visibilities, closure phase, and differential phase. It consists of three telescope channels and an interferometric channel. In this figure an approximate wavelength scale is given on the vertical axis. The presence of $\operatorname{Br} \gamma$ emission is revealed by the bright horizontal line, crossing the three photometric and interferometric channels.

\subsection{Observations with $A M B E R$}

IRC +10420 was observed in visitor mode using VLTI+AMBER on the nights of 14th and 15th June 2006. The observations were performed in the UT1-UT2-UT3 baseline configuration. UT4 was not available during these nights due to technical problems. The telescope configuration delivered projected baselines between $\sim 40$ and $\sim 70 \mathrm{~m}$ and a PA coverage of about $20^{\circ}$ (see Table 1). The AMBER instrument was set-up in the medium spectral resolution mode, giving a 
Table 1. Predicted and measured continuum visibilities for IRC +10420 and the calibrator star HR 7648 . IRC +10420 has an observed upper limit to its diameter of 3 mas (Monnier et al. 2004), whereas the calibrator has a uniform disk diameter of 1.95 mas (Richichi et al. 2005). Corresponding theoretical visibilities are given in Cols. (2) and (6). The measured raw values of $V_{\text {cont }}^{2}$ in Cols. (3) and (7) correspond to the average continuum value for a SNR frame selection of $20 \%$ (see text), these values are subject to systematic uncertainties. The uncertainties on the raw $V_{\text {cont }}^{2}$ given reflect the internal variation. Column (8) is the calibrated $V_{\text {cont }}^{2}$. Column (9) gives the ratio between the line and continuum (see Fig. 3). Column (10) is the range for $V_{\mathrm{Br} \gamma}$ as derived in Sect. 3.3, taking into account the various uncertainty contributions.

\begin{tabular}{|c|c|c|c|c|c|c|c|c|c|c|}
\hline & $\begin{array}{l}\text { HR7648 } \\
\text { Baseline } \\
\text { (m) } \\
(1)\end{array}$ & $\begin{array}{l}V_{\mathrm{UD}} \\
\text { theo. } \\
(2)\end{array}$ & $\begin{array}{c}\left\langle V_{\text {cont }}^{2}\right\rangle \\
\text { raw } \\
(3)\end{array}$ & $\begin{array}{c}\text { IRC }+10420 \\
\text { Baseline } \\
(\mathrm{m}) \\
(4)\end{array}$ & $\begin{array}{l}\text { PA } \\
\circ \\
(5)\end{array}$ & $\begin{array}{c}V \\
\text { theo. } \\
(6)\end{array}$ & $\begin{array}{c}\left\langle V_{\text {cont }}^{2}\right\rangle \\
\text { raw } \\
(7)\end{array}$ & $\begin{array}{c}\left\langle V_{\text {cont }}^{2}\right\rangle \\
\text { calib. } \\
(8)\end{array}$ & $\begin{array}{c}V_{\mathrm{cont}}^{2} / V_{\mathrm{Br} \gamma}^{2} \\
(9)\end{array}$ & $\begin{array}{l}V_{\mathrm{Br} \gamma} \\
(10)\end{array}$ \\
\hline U1-U2 & 43.9 & 0.96 & $0.111 \pm 0.002$ & 39.8 & 12.8 & $>0.92$ & $0.169 \pm 0.004$ & $1.46 \pm 0.03$ & $1.36 \pm 0.07$ & $0.60-0.74$ \\
\hline U2-U3 & 35.8 & 0.97 & $0.149 \pm 0.003$ & 30.3 & 32.7 & $>0.95$ & $0.210 \pm 0.005$ & $1.37 \pm 0.03$ & $1.20 \pm 0.07$ & $0.74-0.89$ \\
\hline U3-U1 & 78.7 & 0.87 & $0.201 \pm 0.004$ & 69.1 & 21.5 & $>0.76$ & $0.265 \pm 0.005$ & $1.15 \pm 0.03$ & $1.68 \pm 0.06$ & $0.39-0.57$ \\
\hline
\end{tabular}

spectral resolution of 1500 . The spectrum covers a wavelength range between 2.12 and $2.19 \mu \mathrm{m}$, and includes the $\mathrm{Br} \gamma$ hydrogen recombination line. $\mathrm{Br} \gamma$ was reported to be in emission by Oudmaijer et al. (1994) and the size of the emitting region is the goal of the current AMBER observations of IRC +10420 .

On the first night a total of 15 observations of IRC +10420 was secured over a period of one hour under reasonable weather conditions. On the second night 5 observations were secured under bad weather conditions. Each observation with AMBER produces a file that consists of 1000 individual exposures, called frames. Each frame is made up of the individual photometric information for each telescope aperture and the interferometric beam information (the interferogram, see Fig. 1). The integration time per frame was taken to be $50 \mathrm{~ms}$. This choice is limited by the movement of the fringes over the detector by atmospheric turbulence (the so-called jitter), which causes attenuation of the fringe visibility.

In order to perform an absolute calibration of the AMBER visibilities, an interferometric calibrator star was observed 5 times each night. The calibrator star, HR 7648 (SAO 125355), is a $K=2.2$, K5 III star with a uniform disk diameter of $1.95 \pm 0.02$ mas derived from spectrophotometric data (Richichi et al. 2005). The aspro tool ${ }^{1}$ returns a size of $2.056 \pm 0.142$ mas for HR 7648 based on magnitude-colour calibration (such as $V$ vs. $V-K$ ) of angular diameters. The calibrator's size is thus not directly measured using interferometry. HR 7648 was chosen as it is close on the sky to IRC +10420 and expected to remain (nearly) unresolved (see Table 1). The calibrator is considerably brighter than the expected correlated magnitude of the science target ( $60 \%$ of $K=3.6$, Monnier et al. 2004). HR 7648 was observed 5 consecutive times, one hour after the first IRC +10420 observation, with the same exposure time as the target. The observation of the calibration star was done under worse seeing $\left(1.1^{\prime \prime}-1.2^{\prime \prime}\right)$ conditions than the target star $\left(0.8^{\prime \prime}\right)$, which implies better AO correction during the target observations.

All AMBER data were corrected for electronic interference noise using the amdc software by Li Causi et al. (2008), and reduced using amdlib software (versions 1.21 and 2.0 beta) with frame bin size 1, i.e. no averaging over the 1000 frames present in each file. The additional data reduction files, a bad pixel map and a flatfield were taken from the AMBER website (versions of 9 Feb. 2006). Visual inspection using the ammyorick environment (e.g. Millour et al. 2007) shows that only the first five IRC +10420 observations actually contain frames with fringes.

\footnotetext{
${ }^{1}$ Aspro is a java applet for the preparation and simulation of VLTI observations; see http://www .mariotti .fr/aspro_page.htm
}

A measure of successfully interfering the three telescope beams and the recording of fringes is provided by the amdlib package parameter signal-to-noise ratio (SNR). The expression for this parameter can be found in Eq. (20) of Tatulli et al. (2007b). It is based on the weighted summing of the correlated flux over all spectral channels per interferogram. There is a strong need to apply a data quality (fringe quality) check to AMBER data due to non-stationary vibrations in the interferometer infrastructure, which are uncontrollable at present. The currently recommended approach regarding these data quality degrading effects is to make a frame selection based on the SNR criterion, and subsequently disregarding low SNR frames up to a certain cut-off (e.g. see Tatulli et al. 2007a). The SNR values calculated by amdlib for these observations range between 1 and 4 . The other ten observations of the first night suffer from very low fluxes, hardly rising above the detector noise level. The five calibrator observations are all of relatively good quality, with some frame SNR values reaching 10. Observations taken during the second night are also of low quality. The results presented in this paper are therefore based on the first five observations of both IRC +10420 and HR 7648 taken during the first night.

Practical experience has led to the compromise to choosing the best $20 \%$ of the frames during the frame selection process (Tatulli et al. 2007a). This $20 \%$ SNR cut-off was chosen only because of the consistent AMBER visibility results it has provided (see e.g. Malbet et al. 2007). In our case, the $20 \%$ selection for calibrator and target would lead to unphysical calibrated target continuum visibilities larger than one $\left(\left\langle V_{\text {cont }}^{2}\right\rangle\right.$ in Col. (8) of Table 1). However, we shall show in the next subsection that frame selection does not seriously affect the relative AMBER visibilities.

The current quality of AMBER data limits the ability to perform an absolute calibration of the continuum visibilities. Instead, we will use the results from previous high-angular resolution studies in the literature. In Table 1, we quote an upper limit to the diameter of IRC +10420 's continuum emitting region of 3 mas based on Keck Interferometer observations in the $K$-band (Monnier et al. 2004). The corresponding lower limits to the visibility for a uniform disk at each VLTI baseline were computed and are listed as well. We should note that 3 mas is probably a generous size upper limit to the size of the continuuum emitting region if it corresponds to the underlying star only. A simple calculation shows that for a luminosity of IRC +10420 of $25462(\mathrm{~d} / \mathrm{kpc})^{2} L_{\odot}$ (Blöcker et al. 1999) and a mid A spectral type (Oudmaijer et al. 1996), a much smaller stellar diameter of $\sim 0.70$ mas can be expected. This result is independent of distance. If instead the stellar disk of IRC +10420 were equal to the $\sim 3$ mas upper limit, then its spectral type would need to be M-supergiant, which is inconsistent with IRC +10420 's A-type 

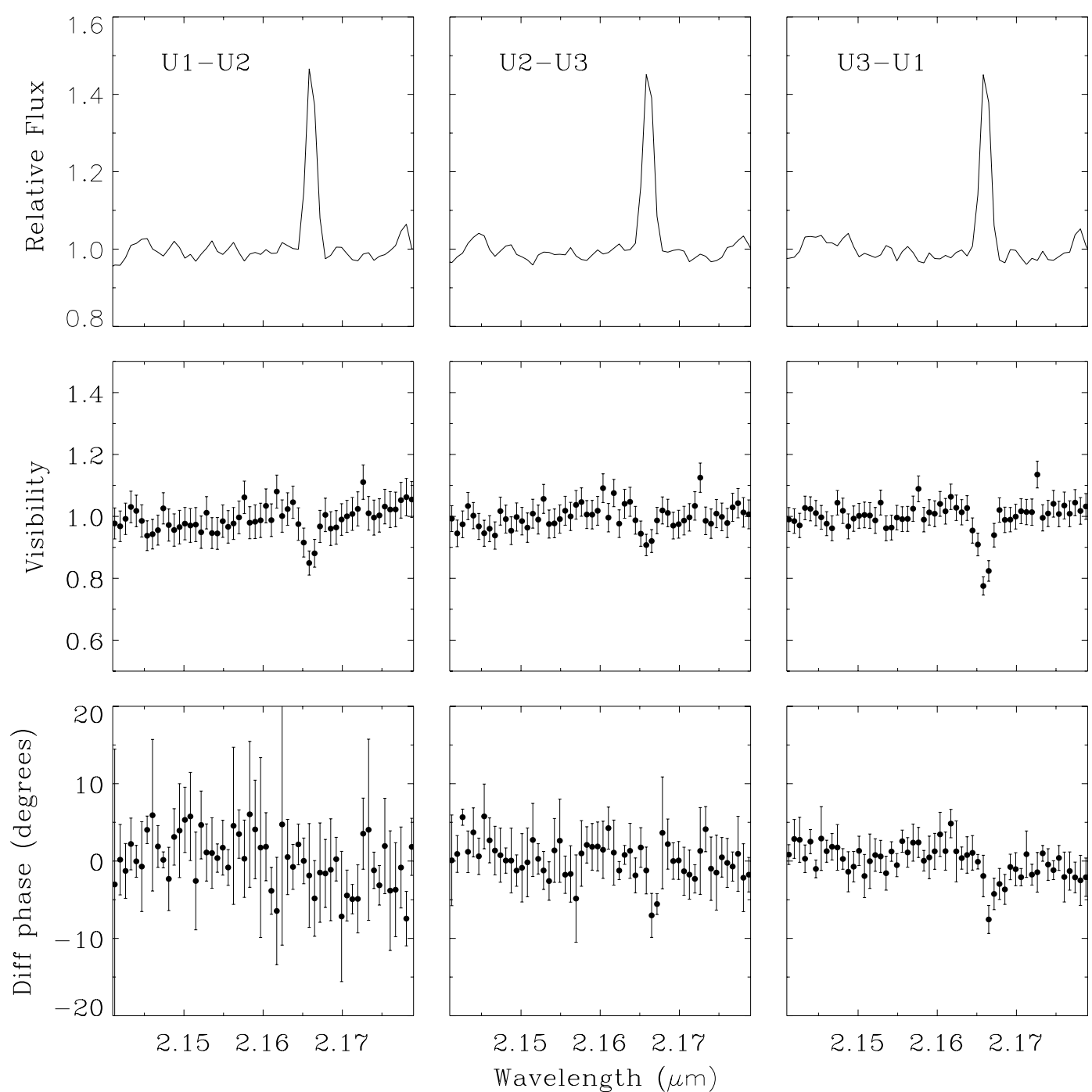

$2.15 \quad 2.16 \quad 2.17$

Fig. 2. AMBER flux (top), visibility (middle) and differential phase (bottom) spectra of IRC +10420 for each of the three VLTI U1-U2-U3 configurations. The flux spectrum is divided by a telluric standard and normalized to one. The continuum visibility is normalised to a value of one near $\mathrm{Br} \gamma$ (see discussion in text). The $\mathrm{Br} \gamma$ emission line has a smaller visibility than the continuum at all three baselines. A phase change is detected for the U3-U1 baseline.

spectrum. As we shall argue below, other contributions to the continuum as seen by AMBER by dust or any bound-free/freefree emission is small.

\section{Results}

\subsection{AMBER flux, visibility, and phase spectra}

The panels of Fig. 2 show the reduced AMBER observables for each UT baseline: the flux (top), visibility (middle) and the differential phase (bottom) spectra. The flux spectra are normalised to one (at Bry). No wavelength calibration has been performed, and the spectra have simply been shifted in wavelength in order to match the emission line with the $\mathrm{Br} \gamma$ rest wavelength. The spectra have been divided by the interferometric calibrator star in order to remove telluric absorption components. The flux spectra show the $\mathrm{Br} \gamma$ transition clearly in emission, as has been reported by three other papers previously, with spectra taken in 1992, 1994, and 2000 (Oudmaijer et al. 1994; Hanson et al. 1996; Humphreys et al. 2002 respectively). The line has an equivalent width $(E W)$ of $-7 \AA$, which is significantly stronger than found in the above mentioned studies. We discuss this difference in the next subsection.

The second row of three panels presents the visibility spectra. The spectra are the average of $20 \%$ best SNR frames. Following the discussion on the calibration of the continuum visibilities, we have set its value to one, i.e. a spatially unresolved continuum (see also next subsection). The important result of these observations is however the smaller visibility in the $\mathrm{Br} \gamma$ emission line as function of baseline. We note that the shortest baseline is the U2-U3 baseline, where the deviation from a spatially unresolved $\mathrm{Br} \gamma$ line is smallest.

It is important to assess the reality of the relative visibility of the $\mathrm{Br} \gamma$ line with respect to the continuum. A good test is to measure its stability as function of the frame selection criteria. In order to do this, we applied a SNR frame selection with an increasing percentage of frames selected and for each selection we calculated the relative visibility of the $\operatorname{Br} \gamma$ line. We started with a minimum percentage of $20 \%$ and increased it in steps of $20 \%$. For each selection we measured the average of the continuum (excluding the $\mathrm{Br} \gamma$ line). The minimum in the $\mathrm{Br} \gamma$ transition was taken to be the minimum of a Gaussian fit to the visibility profile. The fraction $V_{\text {cont }}^{2} / V_{\mathrm{Br} \gamma}^{2}$ as function of percentage of selected 


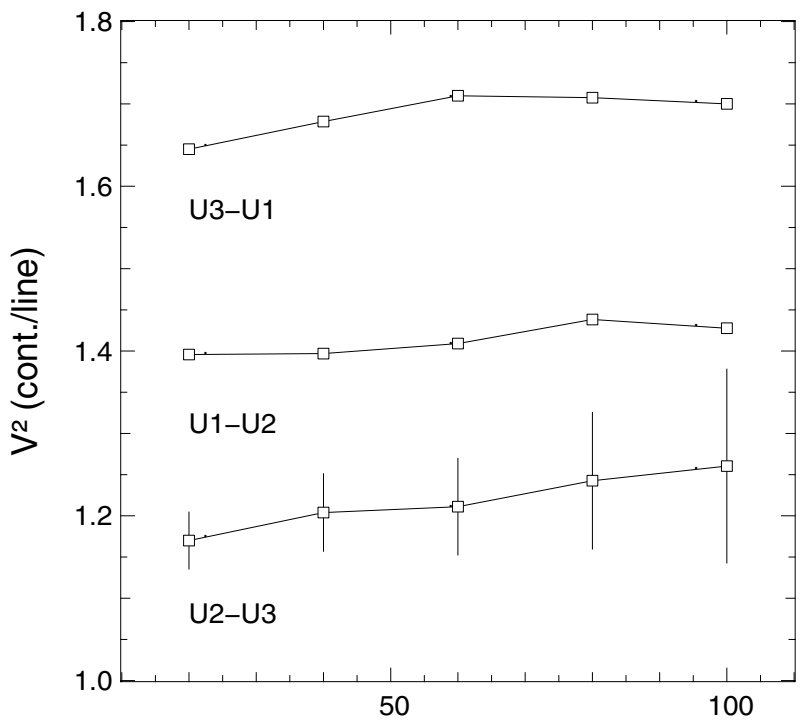

Percentage of frames (\%)

Fig. 3. The ratio of the continuum squared visibility to the Br $\gamma$ squared visibility as function of the number of frames selected.The errorbars indicate the continuum variance per spectrum averaged over the selected frames. For clarity purposes, the errorbars are plotted for one baseline only.

frames is shown in Fig. 3. The figure shows that this fraction slightly increases with the percentage of frames selected. This observed trend is most likely due to a bias towards smaller values of $V^{2}$ which is introduced when more frames are selected. A correct estimate of $V^{2}$ requires a certain level for the incoming flux. As Bry is in emission, more line flux is available for the beam combination than in the adjacent continuum. A reasonable estimate for $V_{\mathrm{Br} \gamma}^{2}$ is therefore obtained for a larger fraction of the frames than for the $V_{\text {cont }}^{2}$ estimate. Increasing the number of selected frames will thus bias the $V_{\mathrm{Br} \gamma}^{2}$ less than it does for $V_{\text {cont }}^{2}$, which results in a larger value for their ratio. The errorbars on the measurements in Fig. 2 are representative of the variance of the continuum visibility level, averaged over the number of selected frames. As can be seen in the figure, the strongest relative dependence on frame selection is found for the shortest baseline 2 (U2-U3), for which the relative visibility still only changes at most by 0.1 . We thus conclude that the AMBER relative visibilities of IRC +10420 are stable within the uncertainties listed. The variation of these fractions are taken into account in the uncertainty in the ratio between line and continuum as quoted in Table 1.

Finally, the last row of panels in Fig. 2 shows the differential phase for each baseline. The differential phase is an approximate measure for the spatial offset of the photocentre on the projected baseline at a wavelength relative to some reference wavelength. The data show no change in differential phase over the $\mathrm{Br} \gamma$ line on the first two baselines, although perhaps a trace is present on the second baseline. A phase change of 5 to 10 degrees in the $\mathrm{Br} \gamma$ line with respect to the continuum is detected on the third baseline. This constitutes an offset of the $\mathrm{Br} \gamma$ photocentre of about 0.1 mas, and will be discussed in Sect. 3.3. The fourth AMBER observable, the closure phase, shows a random white noise scatter with an $\mathrm{rms}$ of $25^{\circ}$ around $0^{\circ}$ (for $20 \%$ best SNR frame selection), without any trace of a signal around the $\mathrm{Br} \gamma$ transition. It suggests that within the errors the line emitting region is centro-symmetric on the sky.

\subsection{Size scales of the dusty CS environment}

The total $K$-band emission of IRC +10420 appears to consist of almost equal contributions by the photosphere and the hot CS dust (Oudmaijer et al. 1996). They find a good SED fit for a star surrounded by two dust shells at distances larger than 86 stellar radii (30 mas). This is also found by Blöcker et al. (1999) whose interferometric observations resolve $\sim 40 \%$ of the $K$-band emission. Their modelling of both the SED and visibilities reveals two dust shells with a minimum radius of $\sim 35$ mas (see also Sect. 1). The $60 \%$ flux component remains unresolved even for baselines up to $36 \mathrm{~m}$ as probed with IOTA (Monnier et al. 2004). Evidently, most of the $K$-band excess corresponds to this dust component, leaving at most $10 \%$ of the (total) continuum flux due to another source. This could be either dust or gas emission located much closer to the star and unresolved by IOTA. The relatively large $E W(\mathrm{Br} \gamma)=-7 \AA$ in the AMBER flux spectra is consistent with the idea that a $40 \%$ reduction of the $K$-band continuum is due to a field of view effect rather than to variable $\operatorname{Br} \gamma$ emission. Previous observations reported $E W(\mathrm{Br} \gamma)$ of $-1.2 \AA$ (Oudmaijer et al. 1994) and $-4 \AA$ (Humphreys et al. 2002). The question is how much the $\operatorname{Br} \gamma$ emission has varied between the 2000 spectrum by Humphreys et al. and the AMBER spectrum of 2006. An as yet unpublished spectrum taken by our group at the NTT with the SOFI imaging spectrograph in 2005 , has an $E W(\mathrm{Br} \gamma)$ of $-4.5 \AA$, i.e. a small change with respect to 2000. This is in line with $E W(\mathrm{H} \alpha)$ that varied relatively little over that same period (Patel et al. 2008). Thus assuming that there was no change in $\mathrm{Br} \gamma$ emission, we conclude that $\sim 40 \%$ less continuum flux must have been observed in the AMBER data, which indicates that most dust is outside AMBER's field of view of 66 mas.

\subsection{Size scale of the Bry emission region}

The size of the $\operatorname{Br} \gamma$ emitting region can be derived from the line visibilities. We estimate the visibility of the $\mathrm{Br} \gamma$ emission component by applying the definition for the total visibility of a multi-component source:

$V_{\mathrm{Br} \gamma+\mathrm{cont}}=\frac{V_{\text {cont }} \times F_{\text {cont }}+V_{\mathrm{Br} \gamma} \times F_{\mathrm{Br} \gamma}}{F_{\text {cont }}+F_{\mathrm{Br} \gamma}}$

We solve for $V_{\mathrm{Br} \gamma}$ using the observed line and continuum flux ratios from the spectrum. However in this procedure there are uncertainties involved regarding the absolute level of $V_{\text {cont }}$ as previously discussed, and the value of $F_{\text {cont }} . F_{\text {cont }}$ is uncertain in the sense that at $\mathrm{Br} \gamma$ the proper underlying photospheric absorption profile needs to be taken into account. We recall that the ratio of $V_{\text {cont }}$ to $V_{\mathrm{Br} \gamma+\text { cont }}$ is stable as shown in Sect. 3.1, $V_{\text {cont }}$ however could in principle lie between the values corresponding to the 3 mas upper limit from IOTA, and the fully unresolved case. Equation (1) is valid if there does not exist a differential phase between line and continuum (see e.g. Weigelt et al. 2007). The effect of the small differential phase on the line visibility detected in U3-U1 is negligible however when compared to other uncertainties that we will discuss next.

Let us first estimate the value for $F_{\text {cont }}$ in Eq. (1) that needs to correspond to the photospheric absorption feature. We estimate the absorption feature from the spectral type of IRC +10420 , viz. mid A-type. We introduce into the AMBER spectrum a Gaussian absorption profile with $E W$ s of 5 and $6 \AA$ with a $F W H M$ similar to the emission line. This $E W$ is about the average for A-type Ia supergiants as reported in Hanson et al. (1996). 


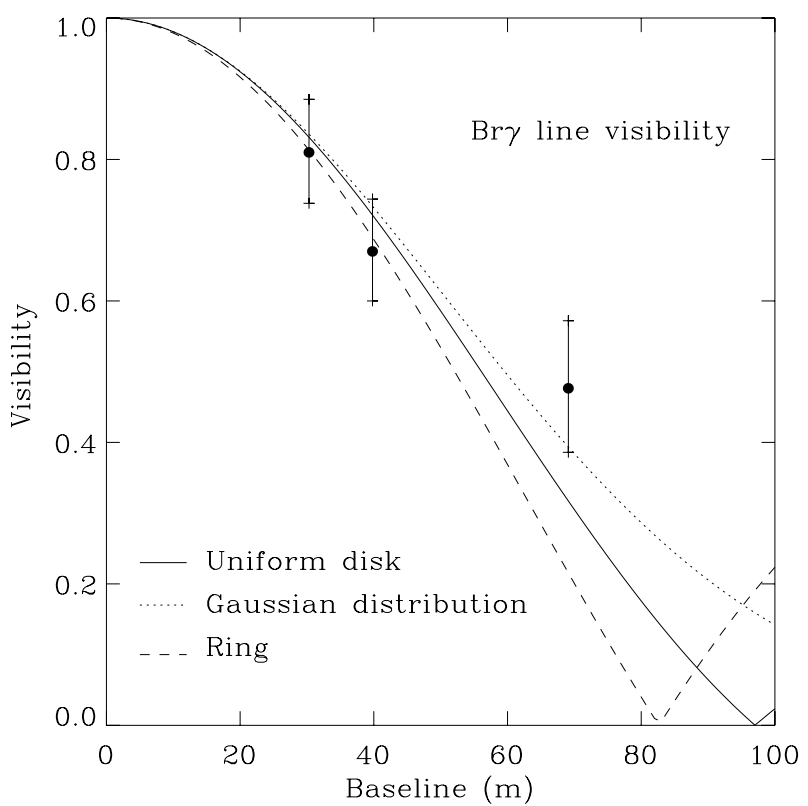

Fig. 4. The final visibilities of the $\mathrm{Br} \gamma$ emitting region. The range in values is determined by the uncertainty in photospheric absorption, the absolute continuum visibility, and the error on the relative visibility of the line. Three simple, symmetric models have been fit to the data. The Gaussian model fits with a FWHM of 3.3 mas.

The ratio of $F_{\mathrm{Br} \gamma}$ to the adjacent continuum is 1.48 , whereas the ratio of $F_{\mathrm{Br} \gamma}$ to the minimum flux level of the photospheric absorption profile is between 1.97 and 2.08 for an equivalent width of $5 \AA$ and $6 \AA$ respectively. Given the discussed uncertainties in the continuum visibilities, we derive a range of values for the $\operatorname{Br} \gamma$ visibility at each baseline. These values are presented in Fig. 4 and listed in Table 1.

In the figure show the best fits to the $\operatorname{Br} \gamma$ visibilities of three different, simple, geometries, viz. the uniform disk, the Gaussian distribution, and a ring distribution. The uncertainties on the visibilities are dominated by three factors, (1) uncertainty of the absolute calibration of the continuum visibility, between 3 mas and unresolved; (2) uncertainty of $\mathrm{Br} \gamma$ visibilities relative to the continuum (see Table 1 Col. (9)); and (3) uncertainty regarding the underlying photospheric absorption. All these three uncertainties are taken into account in the range of $\mathrm{Br} \gamma$ visibilities plotted in Fig. 4 and listed in Col.(10) in Table 1. The figure clearly demonstrates that the shapes of the visibility curves as function of baseline do not follow the observed visibilities for the cases of a uniform disk and a ring. The Gaussian distribution can reproduce the observations, albeit marginally. The Gaussian distribution of Fig. 4 has a FWHM of 3.3 mas. We thus conclude that the $\mathrm{Br} \gamma$ emitting region on the sky of IRC +10420 takes the shape of a Gaussian-like distribution with a FWHM of $\sim 12 \mathrm{AU}$ ( $d=3.5 \mathrm{kpc}$ ), at least between the measured baseline position angles, i.e. between a PA of $\sim 10^{\circ}$ and $\sim 30^{\circ}$.

\section{Discussion: geometry of the $\mathrm{Br} \gamma$ emission region}

Given the limited PA range of our VLTI observations, the shape of the emitting region can not be further constrained by the visibility measurements alone. Only if the line is optically thick can we infer a geometry using the total $\mathrm{Br} \gamma$ flux. Indeed, in that case, the flux can be approximated by $F_{\mathrm{L}}=B_{v}(v, T) \Delta v\left(\frac{R}{d}\right)^{2}$ (cf. Oudmaijer et al. 1994; see also Grundstrom \& Gies 2006), with $B_{v}$ the Planck function, $\Delta v$ the linewidth, unresolved in our data but measured to have a $F W H M$ of $64 \mathrm{~km} \mathrm{~s}^{-1}$ by Oudmaijer et al. (1994) and only slightly broader in Humphreys et al. (2002), $R$ represents the radius of the emitting surface if it is circular, and $d$ is the distance. For completeness, we note that the line optical depth does not affect our determination of the size of the $\mathrm{Br} \gamma$ emitting region described in the previous section.

Oudmaijer et al. (1994) present the dereddened linefluxes of several optical and near-infrared hydrogen recombination lines and demonstrate that the line ratios deviate significantly from case B. For example, IRC +10420 shows $F_{\mathrm{Br} \gamma} \sim F_{\mathrm{Br} \alpha}$, a line ratio that indicates that $\mathrm{Br} \alpha$ is optically thick (case $\mathrm{B}$ predicts a ratio of 0.33). This would not necessarily imply that $\mathrm{Br} \gamma$ is optically thick of course, but we note that the models by Simon et al. (1983) indicate that $\mathrm{Br} \gamma$ is almost as strong as $\operatorname{Br} \alpha$ for a very large range of optical depths for both $\mathrm{Br} \gamma$ and $\mathrm{Br} \alpha$. As both $\mathrm{H} \alpha$ and the $\mathrm{Br} \gamma$ line have become stronger over the years (see Patel et al. 2008, and above respectively), we conclude that it is reasonable to assume the line to be optically thick.

Inspection of the above equation immediately indicates that we can directly compare the size of the emitting region with that of the star emitting at the same wavelength by taking the ratio

$$
\left(\frac{F_{\mathrm{L}}}{F_{*}}\right)=\left(\frac{B_{v}\left(v, T_{\mathrm{L}}\right)}{B_{v}\left(v, T_{*}\right)} \frac{A_{\mathrm{L}}}{A_{*}}\right) .
$$

With $T_{\mathrm{L}}, T_{*}, A_{\mathrm{L}}, A_{*}$, the temperature and the projected surface area of the line emitting region and the star respectively. In the AMBER data, taking into account filling in of the photospheric absorption profile by the line emission, we find that the line and stellar continuum have a roughly equal contribution to the total flux, i.e. $F_{\mathrm{L}} \sim F_{*}$. Assuming a stellar temperature of $8500 \mathrm{~K}$ and a temperature of the ionized region of $5000 \mathrm{~K}$, we can then use Eq. (2) to compute the ratio of line emitting surface area and the stellar disk, which turns out to be 1.7. If we take the same temperature for the star as for the line emitting region, we find a lower limit to the ratio of 1 . So, the flux spectrum indicates that an optically thick line emitting area would be less than twice the size of the stellar photosphere.

How does this compare with the results from the AMBER interferometry? We recall that the Gaussian fit to the visibility of the resolved $\mathrm{Br} \gamma$ line gave a FWHM for the $\mathrm{Br} \gamma$ line emitting area at our projected baseline of 3.3 mas. This diameter is 5 times larger than the stellar diameter as derived from spectral energy distribution considerations and even larger than the upper limit of 3 mas found by Monnier et al. Hence, if the optically thick Br $\gamma$ came from a circular region on the sky, then based on the observed and inferred size scales of the line emitting region and the star respectively, we would predict the line flux to be at least an order of magnitude larger than is actually observed. This apparent contradiction between observed and expected line fluxes can most straightforwardly be explained if the line emitting surface is not circular, but has an asymmetric, elongated, appearance, with the measured size scale along the major axis.

Such an apparent disparity is not unusual, and is a well documented property of Be stars. Their, optically thick, $\mathrm{H} \alpha$ lines are known to originate from a disk and the line fluxes are also comparable to the continuum flux, implying small emitting areas. However, in the cases where the emission is resolved, the disks' major axes are much larger than the unresolved stars. Indeed, the effect is so general that the $E W(\mathrm{H} \alpha)$ can be used to estimate the extension of the disks (see e.g. Grundstrom \& Gies 2006).

In summary, the $\mathrm{Br} \gamma$ line visibilities indicate that AMBER resolves the line emitting region, and we derive a corresponding size of $5 R_{*}$. The region produces a much larger 
line-to-continuum flux ratio than observed in the AMBER flux spectrum, under the assumption of optically thick emission. From this discrepancy one may infer that the $\operatorname{Br} \gamma$ emitting region is actually elongated on the sky along the North-Eastern direction, with a PA of about $20^{\circ}$. A caveat is of course that for an optically thin $\operatorname{Br} \gamma$ line no conclusions can be made regarding the geometry of the emitting region.

That the circumstellar material of IRC +10420 exhibits a North-Eastern extension at larger scales than probed here may not be a coincidence. The outer reflection nebulosity in the HST images of Humphreys et al. (1997) is elongated along a PA of $33^{\circ}$, while Davies et al. (2007) find from a study of the reflected optical spectrum off the dust, that the $\mathrm{H} \alpha$ emission has an axis of symmetry aligned with the extended reflection nebula. However, their data do not allow any conclusions to be drawn about whether the $\mathrm{H} \alpha$ emission is elongated along or perpendicular to the long axis of the dusty reflection nebulosity.

The open question is what the nature of the wind is, is the ionized material located in a bi-polar flow type of geometry or in an edge-on disk? Pending velocity resolved high resolution imaging of the recombination lines, we restrict ourselves to the following. The only known velocity information about the large shell around the object is ambiguous. On the one hand Nedoluha $\&$ Bowers (1992) find from their high resolution imaging in the $\mathrm{OH}$ masers that the Northern part is tilted toward the observer and the Southern part moves away. This picture would favour a bi-polar geometry, as the velocity difference between the northern and southern components is too large to be realistically explained by Keplerian motions. On the other hand, Castro-Carrizo et al. (2007) find in their sub-arcsecond imaging of the CO rotational line emission a largely symmetric appearance, but, intriguingly, present a southern component approaching the observer, in contrast to Nedoluha \& Bowers data. Clearly, the nature of even the large scale structure of the circumstellar data is not settled yet.

\section{Conclusions}

We have presented VLTI/AMBER observations in the $K$-band of the post-Red Supergiant transition object IRC +10420 , with the aim to spatially resolve the $\mathrm{Br} \gamma$ emission region. The observations were done with three UT telescopes and consist of intermediate spectral resolution spectra of the coherent and incoherent flux of IRC +10420 within the AMBER field of view of 66 mas and with an angular resolution of 6.5 mas. The telescope configuration probed a range of $20^{\circ}$ in position angle. The conclusions of this study are as follows.

- The AMBER visibility spectra show a decreased Br $\gamma$ line visibility for all three baselines which indicates that the line emitting region has been resolved. The continuum visibilities are not constrained by this dataset. We fit the Br $\gamma$ line visibilities with simple intensity distributions, and find that a Gaussian distribution with a FWHM of 3.3 mas best fits the data. The derived diameter corresponds to a distance of approximately $5 R_{*}$ from the central star.

- The AMBER differential phase spectra show a change in phase for the U3-U1 baseline at the Br $\gamma$ transition.

This could indicate an offset of the photocentre projected on this baseline. Further measurements at different PAs will help to characterize the nature of this signal.
- The AMBER flux spectra show the $\mathrm{Br} \gamma$ line of IRC +10420 in emission.

It is stronger than reported in two previous studies. We argue that this is due to the fact that most of the $K$-band excess emission due to hot dust is located outside the AMBER field of view. This result is consistent with the speckleinterferometric results presented previously by Blöcker et al. (1999), who find the inner most dust sphere to have a diameter of 70 mas.

We have speculated on the geometry of the $\mathrm{Br} \gamma$ emitting region, concluding that it would be elongated along the interferometer baseline if the emission is optically thick. Optically thick emission seems to be consistent with observed line ratios. Additional measurements at different baseline position angles than the ones presented here will help in further constraining the processes that shape the environment of this massive star in transition to its final fate.

Acknowledgements. R.D.O. is grateful for the support from the Leverhulme Trust for awarding a Research Fellowship. We would like to thank Ben Davies for useful discussions, and Andrew Clarke for communicating his results on the near-infrared spectrum. This manuscript benefited from insightful comments by an anonymous referee.

\section{References}

Blöcker, T., Balega, Y., Hofmann, K.-H., et al. 1999, A\&A, 348, 805 Castro-Carrizo, A., Quintana-Lacaci, G., Bujarrabal, V., Neri, R., \& Alcolea, J. 2007, A\&A, 465, 457

Davies, B., Oudmaijer, R. D., \& Sahu, K. C. 2007, ApJ, 671, 2059

Dwarkadas, V. V., \& Owocki, S. P. 2002, ApJ, 581, 1337

Grundstrom, E. D., \& Gies, D. R. 2006, ApJ, 651, L53

Hanson, M. M., Conti, P. S., \& Rieke, M. J. 1996, ApJS, 107, 281

Heger, A., \& Langer, N. 1998, A\&A, 334, 210

Humphreys, R. M., Smith, N., Davidson, K., et al. 1997, AJ, 114, 2778

Humphreys, R. M., Davidson, K., \& Smith, N. 2002, AJ, 124, 1026

Jones, T. J., Humphreys, R. M., Gehrz, R. D., et al. 1993, ApJ, 411, 323

Kastner, J. H., \& Weintraub, D. A. 1995, ApJ, 452, 833

Klochkova, V. G., Yushkin, M. V., Chentsov, E. L., \& Panchuk, V. E. 2002, Astron. Rep., 46, 139

Li Causi, G., Antoniucci, S., \& Tatulli, E. 2008, A\&A, 479, 589

Maeder, A. 2002, A\&A, 392, 575

Malbet, F., Benisty, M., de Wit, W.-J., et al. 2007, A\&A, 464, 43

Mellema, G. 1997, A\&A, 321, L29

Millour, F., Petrov, R., Malbet, F., et al. 2007, ArXiv e-prints, 705

Monnier, J. D., Millan-Gabet, R., Tuthill, P. G., et al. 2004, ApJ, 605, 436

Nedoluha, G. E., \& Bowers, P. F. 1992, ApJ, 392, 249

Nota, A., \& Clampin, M. 1997, in Luminous Blue Variables: Massive Stars in Transition, ed. A. Nota, \& H. Lamers, ASP Conf. Ser., 120, 303

Nota, A., Livio, M., Clampin, M., \& Schulte-Ladbeck, R. 1995, ApJ, 448, 788

Oudmaijer, R. D., Geballe, T. R., Waters, L. B. F. M., \& Sahu, K. C. 1994, A\&A, 281, L33

Oudmaijer, R. D., Groenewegen, M. A. T., Matthews, H. E., Blommaert, J. A. D. L., \& Sahu, K. C. 1996, MNRAS, 280, 1062

Oudmaijer, R. D., Davies, B., de Wit, W., \& Patel, M. 2008, in Biggest, Baddest, Coolest stars, ed. D. Luttermoser, B. Smith, \& R. Stencel, ASP Conf. Ser., in press

Patel, M., Oudmaijer, R. D., \& Vink, J. S. 2008, MNRAS, submitted

Petrov, R. G., Malbet, F., Weigelt, G., et al. 2007, A\&A, 464, 1

Richichi, A., Percheron, I., \& Khristoforova, M. 2005, A\&A, 431, 773

Simon, M., Felli, M., Massi, M., Cassar, L., \& Fischer, J. 1983, ApJ, 266, 623

Tatulli, E., Isella, A., Natta, A., et al. 2007a, A\&A, 464, 55

Tatulli, E., Millour, F., Chelli, A., et al. 2007b, A\&A, 464, 29

van der Sluys, M. V., \& Lamers, H. J. G. L. M. 2003, A\&A, 398, 181 